\title{
MAGIX is a differentially expressed gene in brain metastatic human breast cancer.
}

Shahan Mamoor, $\mathrm{MS}^{1}$

1shahanmamoor@gmail.com

East Islip, NY USA

Metastasis to the brain is a clinical problem in patients with breast cancer ${ }^{1-3}$. We mined published microarray data ${ }^{4,5}$ to compare primary and metastatic tumor transcriptomes for the discovery of genes associated with brain metastasis in humans with metastatic breast cancer. We found that the MAGI family member, X-linked, encoded by MAGIX, was among the genes whose expression was most different in the brain metastases of patients with metastatic breast cancer as compared to primary tumors of the breast. MAGIX mRNA was present at increased quantities in brain metastatic tissues as compared to primary tumors of the breast. Expression of MAGIX in primary tumors was significantly correlated with patient recurrence-free survival. Modulation of MAGIX expression may be relevant to the biology by which tumor cells metastasize from the breast to the brain in humans with metastatic breast cancer.

Keywords: breast cancer, metastasis, brain metastases, central nervous system metastases, MAGI family member, X-linked, MAGIX, systems biology of breast cancer, targeted therapeutics in breast cancer. 
One report described a $34 \%$ incidence of central nervous system metastases in patients

\section{$\underline{\text { Methods }}$} compared administration of either neratinib or trastuzumab in conjunction with paclitaxel demonstrated that in a randomized, controlled setting, in breast cancer patients treated with neratinib, not only was the incidence of central nervous system recurrence significantly lower, the time to central nervous system metastasis was significantly delayed as compared to patients administered trastuzumab ${ }^{6}$. The alarmingly high rate of central nervous system metastasis described, as well as data, both anecdotal ${ }^{2}$ and from a randomized, controlled setting ${ }^{6}$ illustrating that treatment with trastuzumab may be associated with these events demands an enhanced understanding of the transcriptional makeup of brain metastatic tissues to support identification of therapeutic targets, whether they are treatment related or not. We performed a global comparative analysis of primary and metastatic tumors in patients with brain metastatic breast cancer ${ }^{4,5}$. We discovered differential and increased expression of the gene encoding theMAGI family member, $\mathrm{X}$-linked, MAGIX, in brain metastatic tissues of patients with metastatic breast cancer.

We used datasets GSE $10893^{4}$ and GSE $125989^{5}$ for this global differential gene expression analysis of brain metastatic breast cancer in conjunction with GEO2R. GSE10893 was generated using Agilent-011521 Human 1A Microarray G4110A technology with $n=11$ primary breast tumors and $n=3$ brain metastases from patients with breast cancer; analysis was performed using platform GPL885. GSE125989 was generated using Affymetrix Human Genome U133A 2.0 array technology with $n=16$ primary breast tumors and $n=16$ brain metastases from patients with breast cancer; the samples utilized by investigators for this microarray dataset were paired, matched tumor and metastasis samples; analysis was performed using platform GPL571. The Benjamini and Hochberg method of $p$-value adjustment was used for ranking of differential expression but raw $p$-values were used to assess statistical significance of global differential expression. Log-transformation of data was auto-detected, and the NCBI generated category of platform annotation was used. A statistical test was performed to evaluate whether MAGIX gene expression was significantly different between primary tumors of the breast and brain metastases in humans with breast cancer using a two-tailed t-test. For Kaplan-Meier survival analysis, we used the Kaplan-Meier plotter online tool for correlation of MAGIX mRNA expression levels with recurrence-free survival in $n=3951$ breast cancer patients.

\section{$\underline{\text { Results }}$}

We performed global comparative transcriptome analysis of metastatic tumor tissues of patients with metastatic breast cancer using published microarray data ${ }^{4,5}$ to describe the transcriptional landscape of brain metastasis in human breast cancer in an unbiased fashion and for the discovery of novel therapeutic targets.

MAGIX is differentially expressed in the brain metastases of patients with brain metastatic breast cancer.

Through blind, systems-level analysis of published microarray data ${ }^{4}$, we identified the MAGI family member, X-linked, encoded by MAGIX, as a differentially expressed gene in the 
breast metastatic tissues of humans with breast cancer (Table 1). When sorting each of the genes expressed in brain metastases based on significance of difference as compared to primary tumors of the breast in patients with breast cancer, MAGIX ranked 119 out of 17418 total transcripts (Chart 1), equating to $99.3 \%$ differential expression. Differential expression of MAGIX in the brain metastases of patients with metastatic breast cancer was statistically significant (Chart 1; $p=2.36 \mathrm{E}-04)$.

We queried a second microarray dataset ${ }^{5}$ to attempt to validate transcriptome-wide differential expression of MAGIX in human brain metastatic breast cancer. Again, we identified MAGIX as a differentially expressed gene in the brain metastatic tissues of patients with breast cancer (Chart 2). When sorting each of the genes expressed in brain metastases based on significance of difference as compared to primary tumors of the breast in patients with breast cancer, in this dataset, MAGIX ranked 2538 out of 22277 total transcripts (Chart 2), equating to $88.6 \%$ differential expression. Differential expression of MAGIX in the brain metastases of patients with metastatic breast cancer in this dataset was statistically significant (Chart 2; $p=7.84 \mathrm{E}-02$ ). Thus, differential expression of MAGIX, transcriptome-wide, in the brain metastases of women with metastatic breast cancer was conserved across two independent microarray datasets.

\section{MAGIX is expressed at higher levels in the brain metastases of patients with metastatic} breast cancer.

We obtained exact mRNA expression levels for MAGIX, in primary tumors of the breast and in brain metastasis of patients with brain metastatic breast cancer to determine direction and statistical significance of change in MAGIX expression in brain metastatic tissues. MAGIX was expressed at higher levels in the brain metastases of patients with breast cancer as compared to primary tumors of the breast, and this difference was statistically significant (Figure 1; $p=0.000217)$.

\section{MAGIX expression is significantly correlated with survival outcomes in human breast cancer.}

We performed Kaplan-Meier survival analysis ${ }^{7}$ to evaluate correlation in 3951 breast cancer patients in total, to evaluate whether MAGIX tumor expression was correlated with survival outcomes in breast cancer. We observed a statistically significant correlation between primary tumor expression of MAGIX and recurrence-free survival (RFS) (Figure 2). Patients whose primary tumors expressed low levels of MAGIX possessed median RFS of 40.44 months, while patients whose tumors expressed high levels of MAGIX possessed median RFS of 58 months. This difference in OS based on MAGIX tumor expression in breast cancer was statistically significant (Figure 2, Chart 3; logrank $p$-value: 9.3e-5; hazard ratio: 0.8 (0.72-0.9)).

Thus, by mining published microarray data ${ }^{4,5}$ in an unbiased and systematic fashion, we identified the MAGI family member, X-linked, MAGIX, as among the genes whose expression was most different, transcriptome-wide, in the brain metastases of patients with breast cancer 
when compared to primary tumors of the breast; we observed significantly increased expression of MAGIX in brain metastases as compared to primary tumors of the breast. Further, we found a significant correlation between MAGIX expression and patient survival outcomes, as recurrence-free survival was significantly higher in patients whose primary tumors expressed higher levels of MAGIX as compared to patients whose primary tumors expressed lower levels of MAGIX.

\section{Discussion}

We provided evidence here that the MAGI family member, X-linked, encoded by MAGIX, is among the genes whose expression is most different in the brain metastases of patients with brain metastatic breast cancer, that messenger RNA for MAGIX is present at significantly increased quantities in the brain metastatic tissues of patients with metastatic breast cancer as compared to primary tumors of the breast and that primary tumor MAGIX expression is significantly correlated with patient survival outcomes in human breast cancer. Evaluation of the effects of genetic depletion of MAGIX in mouse models of metastatic breast cancer on metastasis to the central nervous system is merited. Changes in MAGIX expression may be relevant to the processes by which tumor cells exit the breast, enter the vasculature and/or lymphatics, reside in the lymph nodes, evade immune clearance, breach the blood-brain barrier and colonize the brain. 


\section{References}

1. Lin, N.U., Amiri-Kordestani, L., Palmieri, D., Liewehr, D.J. and Steeg, P.S., 2013. CNS metastases in breast cancer: old challenge, new frontiers.

2. Bendell, J.C., Domchek, S.M., Burstein, H.J., Harris, L., Younger, J., Kuter, I., Bunnell, C., Rue, M., Gelman, R. and Winer, E., 2003. Central nervous system metastases in women who receive trastuzumab-based therapy for metastatic breast carcinoma. Cancer, 97(12), pp.2972-2977.

3. Tsukada, Y., Fouad, A., Pickren, J.W. and Lane, W.W., 1983. Central nervous system metastasis from breast carcinoma autopsy study. Cancer, 52(12), pp.2349-2354.

4. Weigman, V.J., Chao, H.H., Shabalin, A.A., He, X., Parker, J.S., Nordgard, S.H., Grushko, T., Huo, D., Nwachukwu, C., Nobel, A. and Kristensen, V.N., 2012. Basal-like Breast cancer DNA copy number losses identify genes involved in genomic instability, response to therapy, and patient survival. Breast cancer research and treatment, 133(3), pp.865-880.

5. Iwamoto, T., Niikura, N., Ogiya, R., Yasojima, H., Watanabe, K.I., Kanbayashi, C., Tsuneizumi, M., Matsui, A., Fujisawa, T., Iwasa, T. and Shien, T., 2019. Distinct gene expression profiles between primary breast cancers and brain metastases from pair-matched samples. Scientific reports, 9(1), pp.1-8.

6. Awada, A., Colomer, R., Inoue, K., Bondarenko, I., Badwe, R.A., Demetriou, G., Lee, S.C., Mehta, A.O., Kim, S.B., Bachelot, T. and Goswami, C., 2016. Neratinib plus paclitaxel vs trastuzumab plus paclitaxel in previously untreated metastatic ERBB2-positive breast cancer: the NEfERT-T randomized clinical trial. JAMA oncology, 2(12), pp.1557-1564.

7. Györffy, B., Lanczky, A., Eklund, A.C., Denkert, C., Budczies, J., Li, Q. and Szallasi, Z., 2010. An online survival analysis tool to rapidly assess the effect of 22,277 genes on breast cancer prognosis using microarray data of 1,809 patients. Breast cancer research and treatment, 123(3), pp.725-731. 


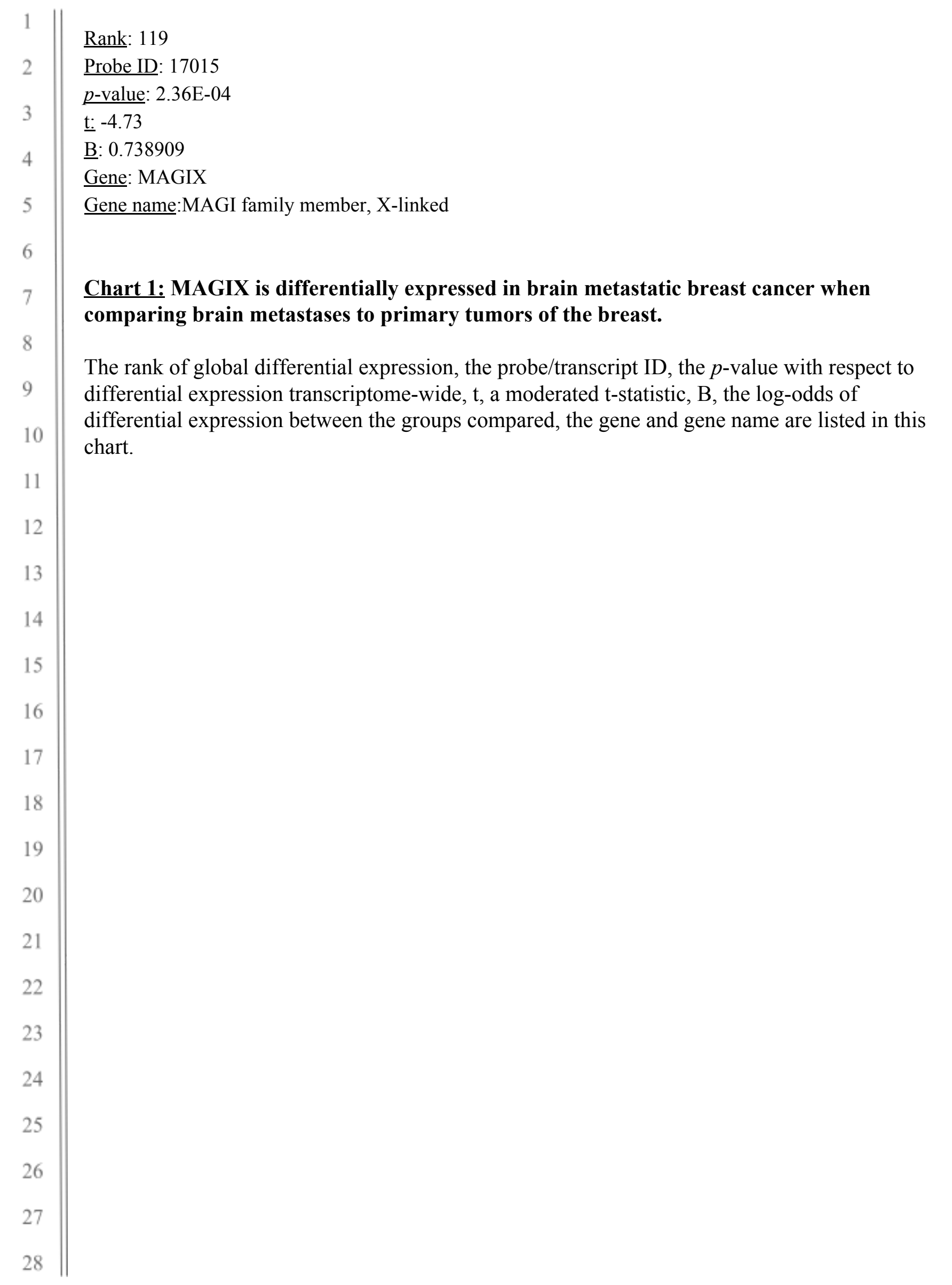


Rank: 2538

Probe ID: 219965 s_at

p-value: $7.84 \mathrm{E}-02$

3 t: 1.8091122

4 B: -4.4898

Gene: MAGIX

5 Gene name: MAGI family member, X-linked

6

Chart 2: MAGIX is differentially expressed in brain metastatic breast cancer when comparing brain metastases to primary tumors of the breast.

The rank of global differential expression, the p-value with respect to differential expression transcriptome-wide, $\mathrm{t}$, a moderated t-statistic, $\mathrm{B}$, the log-odds of differential expression between the groups compared, the gene and gene name are listed in this chart. 


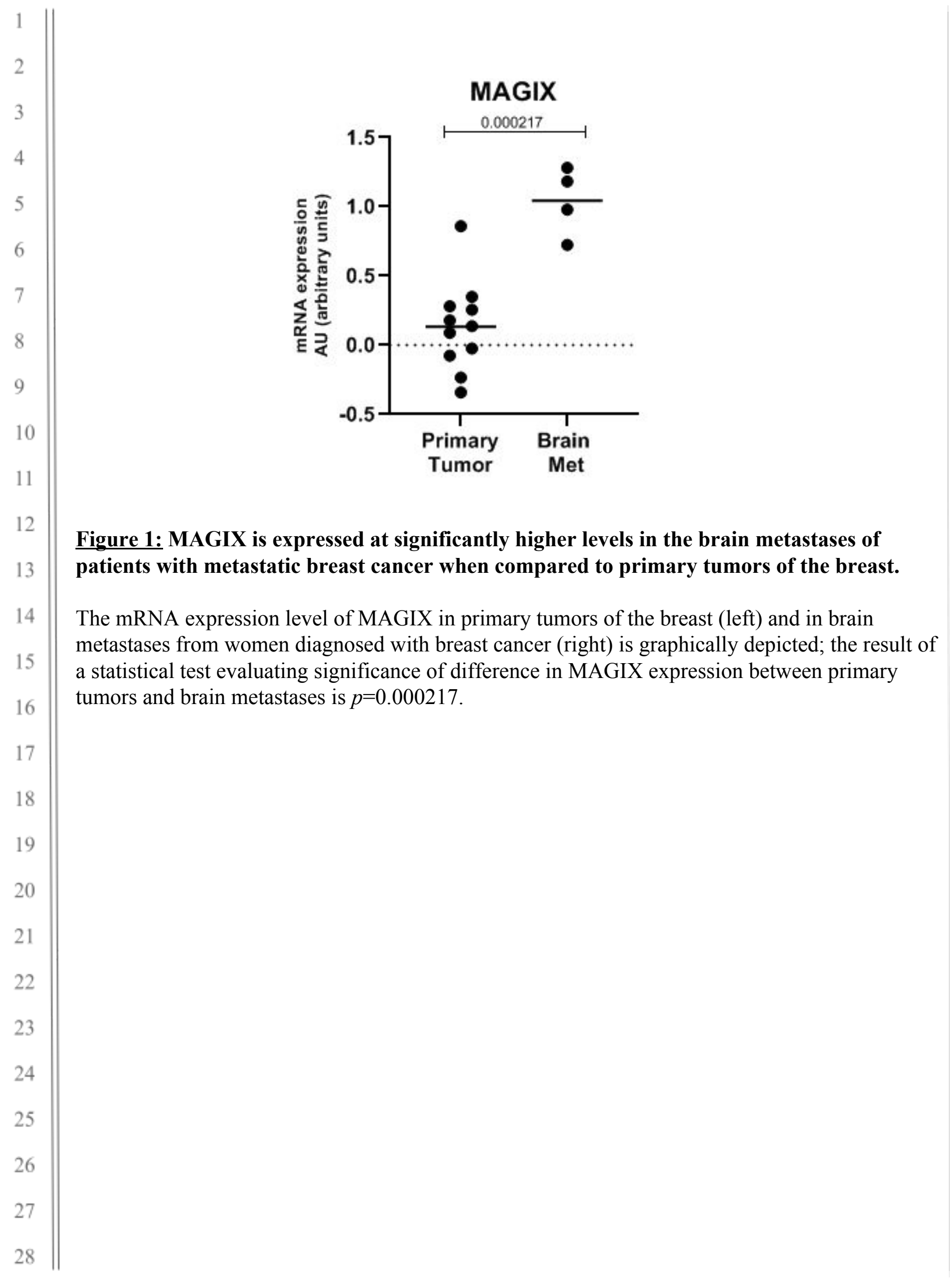




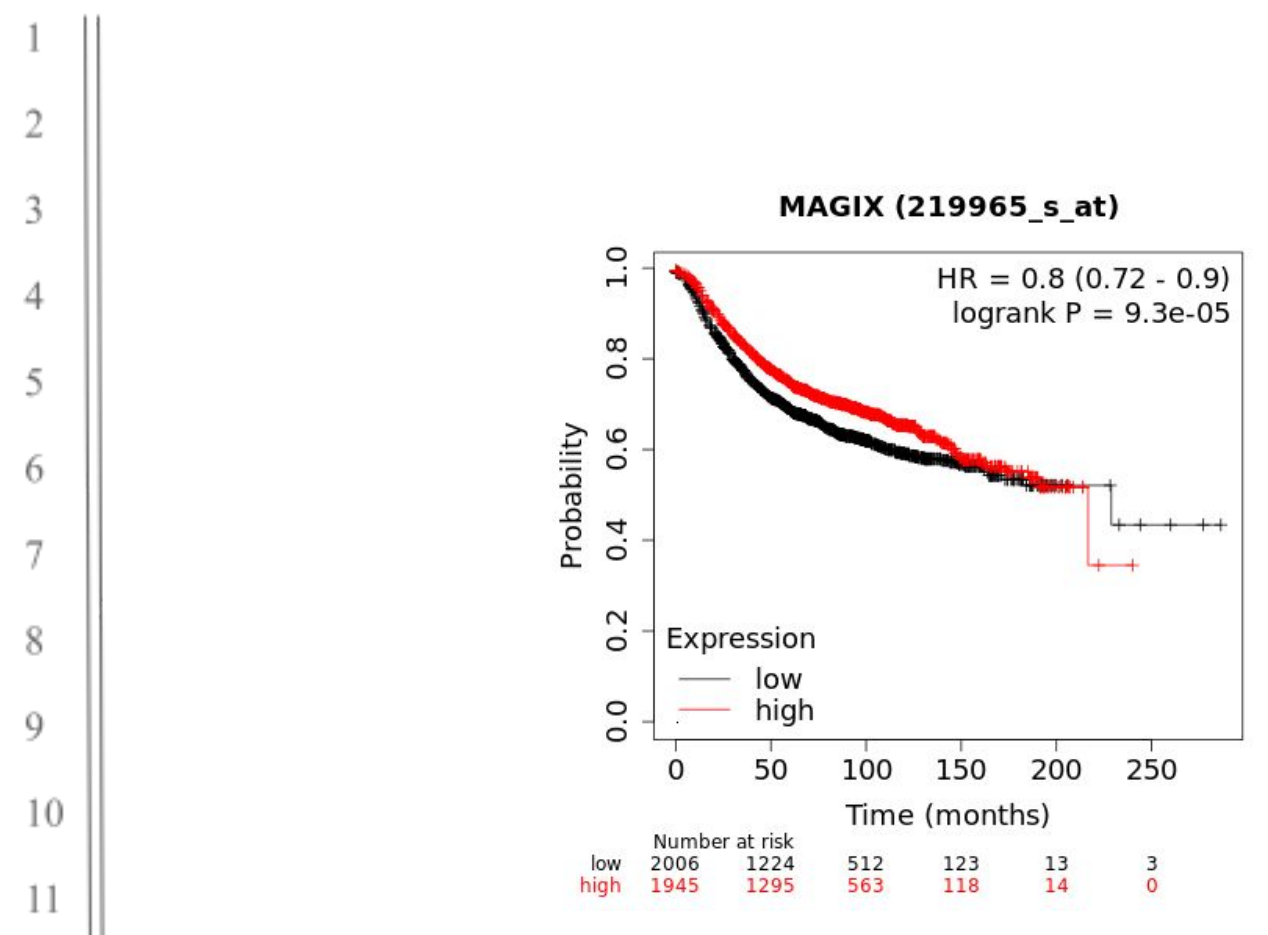

Figure 2: Significant correlation between MAGIX primary tumor expression and recurrence-free survival in patients with breast cancer.

Depicted in this Kaplan-Meier plot is the probability of recurrence-free survival for $n=3951$ total patients stratified into two groups, based on low or high expression of MAGIX in patient primary tumors. The log rank $p$-value denoting statistical significance of difference in recurrence-free survival when comparing the two groups, as well as hazard ratio for this comparison is listed above. Listed below is the number of patients at risk (number of patients alive) per interval, after stratification based on MAGIX expression; in the first interval, number at risk is number of patients alive; in each subsequent interval, number at risk is the number at risk less those who have expired or are censored. 


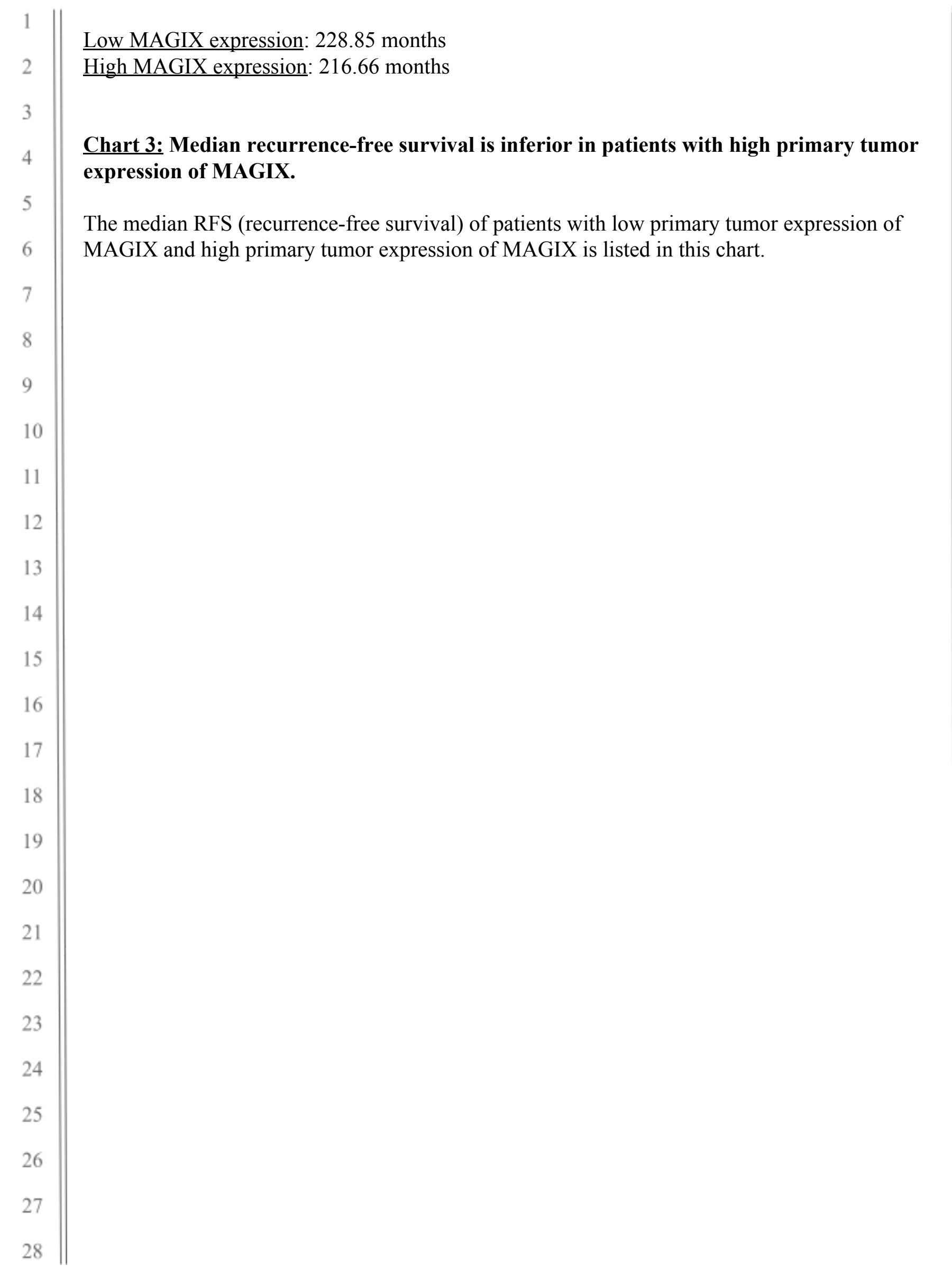

